\title{
Massive intercostal hemorrhage after implantation of an intrapericardial ventricular assist device: a case report
}

\author{
James Etheridge $^{1}$, Ryan Cousins ${ }^{2}$, and Jonathan Philpott ${ }^{3}$ \\ ${ }^{1}$ Brigham and Women's Hospital \\ ${ }^{2}$ Emory University School of Medicine \\ ${ }^{3}$ Eastern Virginia Medical School
}

September 11, 2020

\begin{abstract}
Left ventricular assist devices (LVADs) have become integral to the treatment of advanced heart failure. Surgical bleeding is a known complication of LVAD placement but is most associated with intraperitoneal pump locations. Here we describe a case of massive postoperative hemorrhage secondary to erosion of an intrapericardial LVAD into an intercostal artery with an associated rib fracture.
\end{abstract}

\section{Introduction}

Left ventricular assist devices (LVADs) have become integral to the treatment of advanced heart failure. Surgical bleeding is a common complication of LVAD implantation, with rates reported as high as $60 \%$; the majority of cases require surgical intervention. ${ }^{1,2}$ The intrapericardial design of centripetal flow LVADs may reduce the risk of surgical bleeding by obviating the need for a preperitoneal or intraperitoneal pocket to house the pump. However, recent studies have shown no convincing evidence of this. ${ }^{2,3}$ Here we describe an unusual source of surgical bleeding after intrapericardial LVAD placement caused by erosion into an intercostal artery.

\section{Case summary}

A 54-year-old man with end-stage ischemic cardiomyopathy was admitted to our center for LVAD placement. Echocardiography revealed a left ventricular ejection fraction of $10 \%$ with an end-diastolic dimension of 8.5 $\mathrm{cm}$. He was approved for a HeartWare HVAD (HeartWare International, Framingham, MA), which was placed in the standard apical position via median sternotomy (Figure 1). A polytetrafluoroethylene (PTFE) membrane was placed over the pump housing and outflow graft, as is our usual practice. Hemostasis was achieved quickly, and no intraoperative complications were noted. Postoperatively, the patient complained of severe left-sided chest pain despite appropriate analgesia. This was attributed to poor pain tolerance and his narcotic dosing was increased. No evidence of trauma or significant effusion was found on serial chest radiographs; however, the pump housing appeared to contact the chest wall in several films.

On postoperative day 4, LVAD flows acutely declined. Perfusion was lost despite aggressive fluid resuscitation but was regained with high-dose inotropic support and a brief period of external cardiac massage. A chest radiograph at that time demonstrated a large left pleural effusion. A chest tube was placed with immediate return of 2,200 $\mathrm{mL}$ of frank blood. He was taken emergently to the operating room for exploration. All anastomoses remained hemostatic; however, a massive hemothorax was appreciated in the left pleural space. On further examination, there was an area of pericardium and PTFE through which the pump housing had eroded (Figure 2). The overlying rib was fractured and denuded of periosteum, and the intercostal 
artery was bleeding briskly. Hemostasis was achieved with electrocautery and the fractured rib segment was removed. The remainder of his postoperative course was uncomplicated and he was discharged home in stable condition one month after his index procedure.

\section{Discussion}

Surgical bleeding after LVAD placement typically occurs at cannulation sites, driveline sites, or pump pockets. ${ }^{4}$ The proponderance for erosion into surrounding structures is well-recognized, but is generally associated with an intraperitoneal pump pockets. Per our review of the literature, no cases of erosion into the chest wall by and intrapericardial LVAD have been reported to date.

In this case, several factors suggest that hemorrhage resulted from sustained contact between the pump housing and the chest wall. Iatrogenic rib fractures from median sternotomies are not uncommon and could certainly lacerate intercostal vessels. However, there was no excessive spreading during this procedure, and postoperative chest radiographs demonstrated no evidence of fracture. External cardiac massage can also result in rib fractures, but the intercostal hemorrhage clearly preceded chest compressions. Rather, we observed obvious erosion of the pump housing through the overlying PTFE membrane and pericardium. This aligned perfectly with the injured segment of the chest wall.

The presence of a rib fracture at this site suggests that the pump housing can exert substantial force on adjacent structures. As such, a diaphragmatic position should be strongly considered in the setting of significant left ventricular dilation or massive cardiomegaly, particularly in a thin patient. If the standard apical position is used, a high index of suspicion must be maintained for chest wall trauma. Ultrasound or two-view chest radiography should be used to assess for injury if there is significant left-sided chest pain, and any evidence of hemodynamic compromise should trigger immediate consideration of surgical bleeding.

\section{Conclusion}

This case highlights a rare but potentially devastating complication of intrapericardial LVAD placement. Fortunately, the patient recovered well and faces no major long-term consequences. However, symptoms of ongoing chest wall trauma went unrecognized for three days, despite radiography demonstrating the pump housing abutting the chest wall. In patients with significant cardiomegally or left ventricular dilation, alternative siting strategies should be considered, and a high index of suspicion should be maintained for chest wall injury if the apical position is used.

\section{Conflict of interest}

Jonathan Philpott is a consultant and paid speaker for Medtronic. The remaining authors certify that they have no conflict of interest with any financial organization regarding the material discussed in the manuscript.

\section{Ethics statement}

Consent for publication was obtained verbally from the patient. Institutional review board approval was not required for the described case.

\section{Author contributions}

All authors contributed equally to the manuscript and read and approved the final version of the manuscript.

\section{References}

1. Miller LW, Pagani FD, Russell SD, et al. Use of a continuous-flow device in patients awaiting heart transplantation. N Engl J Med. 2007;357(9):885-896.

2. Rogers JG, Pagani FD, Tatooles AJ, et al. Intrapericardial Left Ventricular Assist Device for Advanced Heart Failure. N Engl J Med. 2017;376(5):451-460.

3. Sabashnikov A, Mohite PN, Zych B, et al. Outcomes in patients receiving HeartMate II versus HVAD left ventricular assist device as a bridge to transplantation. Transplant Proc. 2014;46(5):1469-1475. 
4. Kilic A, Acker MA, Atluri P. Dealing with surgical left ventricular assist device complications. J Thorac Dis. 2015;7(12):2158-2164.

\section{Legends}

Figure 1.? Postoperative chest radiograph showing the HeartWare HVAD device (HeartWare International, Framingham, MA) in the apical position. The pump housing appears to be closely apposed to the chest wall.

Figure 2.? Internal view of the left hemithorax during exploration. The pump housing has eroded through the PTFE membrane and pericardium (black arrow), and the overlying rib is fractured and denuded of periosteum (white arrow). Bleeding from the intercostal artery has been controlled with electrocautery.

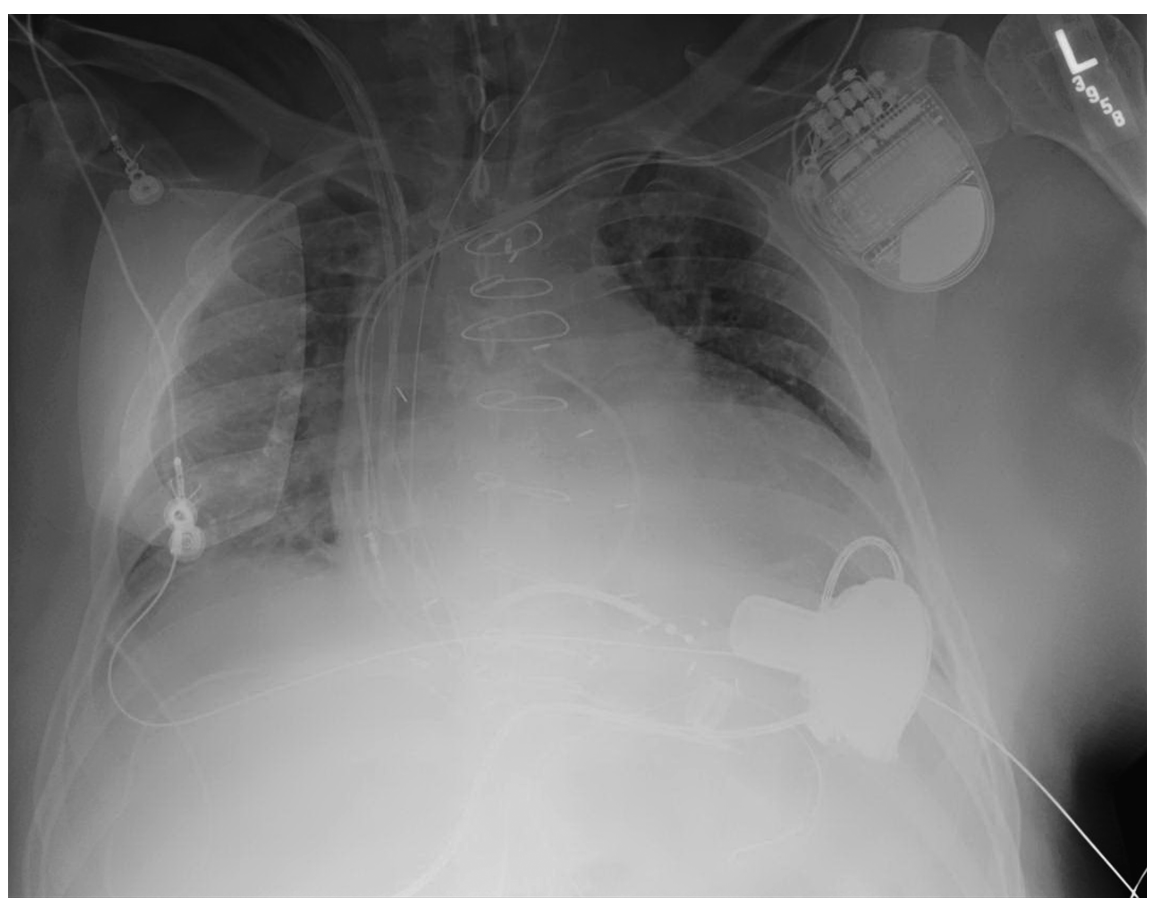




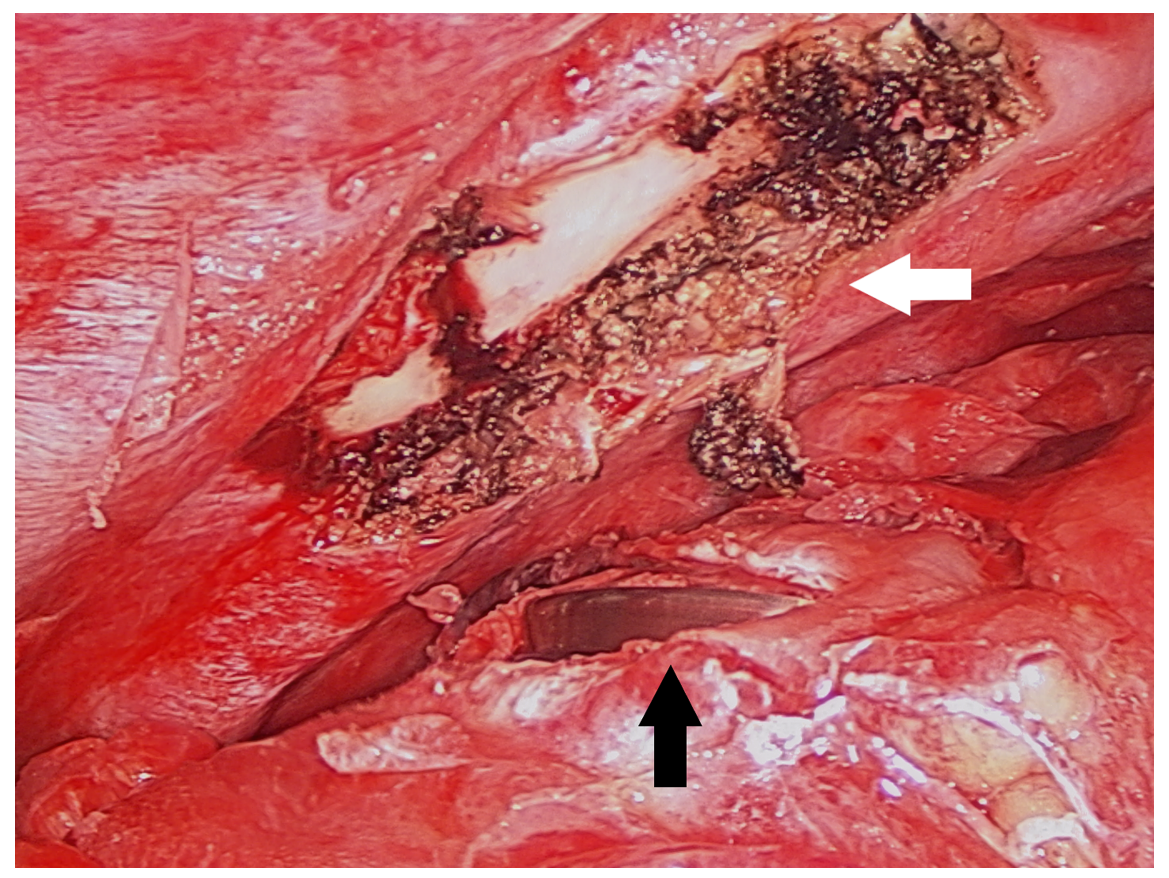

